\title{
Survival of expanded dopaminergic precursors is critical for clinical trials
}

To THE EDITOR-In the August issue of Nature Neuroscience, Studer and coworkers presented an interesting paper describing transplants of expanded mesencephalic precursors in Parkinsonian rats $^{1}$. In keeping with earlier findings ${ }^{2}$, they demonstrate that neuronal precursors from embryonic mesencephalon can be expanded in the presence of basic fibroblast growth factor, and that a significant proportion (about 18\%) of these neuroblasts differentiate into dopaminergic neurons after withdrawal of the growth factor. Studer et al. also uniquely demonstrate that these neurons can survive intracerebral transplantation and reverse a behavioral deficit in hemiparkinsonian rats. Although they suggest that the expansion of mesencephalic dopaminergic neurons can reduce the amount of donor tissue needed in grafting experiments, we feel there is need for caution. Earlier studies have shown that, without any neuroprotective treatment, typically 3000-5000 dopaminergic neurons survive grafting from the mesencephalon of one rat embryo (for example, refs 3,4 ), which is equivalent to a survival rate of around $10 \%$. Studer et al. obtain around 110,000 mesencephalic cells from each embryo, which then undergo a 10 -fold proliferation, similar to that previously reported. Single spheres of differentiating neurons are cultured starting with 200,000 proliferated cells (less than 1/5 of one embryo starting material). Each transplant recipient then received a mean of 6-7 spheres, equivalent to about one embryo starting material, and exhibited a mean of 1221 surviving grafted dopaminergic neurons. Thus, even after the proliferation step, the survival per embryo is clearly less than when transplanting the tissue directly without an intervening proliferation step. Indeed, according to their own calculations they lose as many as $95-97 \%$ of the differentiated dopaminergic neurons in the grafting step. This does not in any way diminish the importance of the findings demonstrating expansion of dopaminergic neurons, but highlights that future studies must attempt to reduce the death of such cells during their differentiation and subsequent transplantation, possibly by the administration of growth factors or antioxidants ${ }^{3-5}$. Only when there is a actual gain in the numbers of surviving dopaminergic neurons from one embryo can such a technique be truly valuable in clinical trials.

\section{Patrik Brundin and \\ Anders Björklund}

Wallenberg Neuroscience Center, Lund

University, Sölvegatan 17, 5-22362

Lund, Sweden

REPLY - We recognize the important contributions made by the Swedish groups in Lund and Stockholm to cell therapy for Parkinson's disease and welcome the opportunity to respond to the letter from Drs. Brundin and Björklund. There are several sites where grafting technology can be optimized: precursor cell expansion, neuronal survival and functional integration in vivo.

Our recently published work ${ }^{1}$ is directed at the first of these issues and makes one specific claim-that functional dopaminergic neurons can be derived from in vitro expanded precursors. There are 30,000 tyrosine hydroxylase-positive $(\mathrm{TH}+)$ neurons in the adult rat substantia nigra ${ }^{6}$. We show that 200,000 precursor-derived TH+ neurons can be obtained per embryo in vitro and that these neurons synthesize and release dopamine in response to depolarization. We also show in vivo that 1,200 $\mathrm{TH}+$ neurons survive from an initial graft of 40,000 $\mathrm{TH}+$ neurons and that these cells lead to functional recovery. Further studies will be needed to compare directly the in vivo survival of dopaminergic neurons derived from expanded precursors with that of primary cell grafts. Historical comparisons are problematic, especially as the studies cited in the above letter ${ }^{3,4}$ analyzed graft survival 1-6 weeks after transplantation, whereas our study focused on more long-term survival (three months). Although several other studies ${ }^{2,7,8}$ also claim that $\mathrm{TH}+$ cells can be obtained in culture, our data demonstrate, with in vitro and in vivo measures, that functional dopaminergic neurons can indeed be derived from expanded precursors.

We agree that improving neuronal survival is an important goal and also urge cautious application of new methods in the clinic. To achieve the clinical goals we all seek, it will be useful to have access to precursor populations for the generation of functional neurons.

\section{Lorenz Studer, Viviane Tabar and Ronald McKay}

Laboratory of M olecular Biology, NINDS, $\mathrm{NIH}, 36$ Convent Drive, Building 36, Room 5A29, Bethesda, M aryland 20892, USA

1. Studer, L., Tabar, V. \& McKay R. D. G. Nature Neurosci. 1, 290-295 (1998).

2. Bouvier, M. M. \& Mytilineou, C. J. Neurosci. 15, 7141-7149 (1995).

3. Zawada, W. M. et al. Brain Res. 786, 96-103 (1998).

4. Nakao, N., Frodl, E. M., Duan, W.-M., Widner, H. \& Brundin, P. Proc. Natl. Acad. Sci. USA 91, 12408-12412 (1994).

5. Rosenblad, C, Martinez-Serrano, A. \& Björklund, A. Neuroscience 75, 979-985 (1996).

6. Rosenthal, A. Neuron 20, 169-172 (1998).

7. Ling, Z. D., Potter, E. D., Lipton, J. W. \& Carvey, P. M. Exp. Neurol. 149, 411-423 (1998).

8. Du, X., Stull, N. D. \& Iacovitti, L. J. Neurosci. 14, 7688-7694 (1994). 